\title{
Scattering of flexural waves by a semi-infinite crack in an elastic plate carrying an electric current
}

\author{
by \\ Jane B. Lawrie \\ Department of Mathematics \\ Brunel University, Uxbridge, Middlesex, UB8 3PH, UK \\ jane.lawrie@brunel.ac.uk \\ and \\ I. David Abrahams \\ School of Mathematics \\ University of Manchester, Manchester M13 9PL, UK \\ i.d.abrahams@manchester.ac.uk
}

\begin{abstract}
Smart structures are components used in engineering applications that are capable of sensing or reacting to their environment in a predictable and desired manner. In addition to carrying mechanical loads, smart structures may alleviate vibration, reduce acoustic noise, change their mechanical properties as required or monitor their own condition. With the latter point in mind, this article examines the scattering of flexural waves by a semi-infinite crack in a non-ferrous thin plate that is subjected to a constant current aligned in the direction of the crack edge. The aim is to investigate whether the current can be used to detect or inhibit the onset of crack growth. The model problem is amenable to exact solution via the Wiener-Hopf technique, which enables an explicit analysis of the bending (and twisting) moment intensity factors at the crack tip, and also the diffracted field. The latter contains an edge wave component, and its amplitude is determined explicitly in terms of the current and incidence angle of the forcing flexural wave. It is further observed that the edge wave phase speed exhibits a dual dependence on frequency and current, resulting in two distinct asymptotic behaviours.
\end{abstract}

\section{Introduction}

Recent advances in non-destructive testing include the implementation of smart structures. In addition to their primary purpose, such components are designed to facilitate or perform at least one more function. A load bearing engineering element may, for example, be designed to allow continual crack diagnosis by the incorporation of an integrated system to record changes in mechanically induced flexural waves. The latter are scattered at a crack interface, thereby providing vital information about the integrity of the structure. Smart structures could detect the scattered vibrations in several ways: for example by measurement of its electrical properties if it is either manufactured using integrated piezoelectric materials or an external electromagnetic field is generated around the body which interacts with the elastic field. Recent research [1] has demonstrated that the presence of such an external field in a thin plate containing a finite length crack has a significant effect on the bending moment intensity factor at the crack tip. Such phenomena could, in principle, be a means not 
only to detect the crack but to control the onset of crack growth within a structure. To date, however, the effect of an electric current on the diffracted field and/or on the amplitude and phase speed of waves localised along the crack faces (edge waves) has received little or no attention.

Konenkov [2] is widely attributed ${ }^{1}$ to have been the first, in 1960, to have demonstrated the existence of a flexural wave guided by the free edge of a semi-infinite isotropic thin elastic plate. Perhaps due to the inaccessibility of his work to western researchers, this result was independently rediscovered some fourteen years later by Sinha [5] and by Thurston and McKenna [6] resulting in two articles appearing in western journals. Such waves, now termed edge waves, have properties analogous to a Rayleigh surface wave on an elastic half-space; that is, they decay exponentially with distance from the edge. Interest in the topic of edge waves picked up in the 1990s and since then a wide variety of results concerning the existence of edge waves have appeared in the literature [7]- [10].

The model problem considered herein comprises an infinite elastic plate with a semi-infinite straight crack. The plate is not subject to fluid-loading but a uniform current flows in the plate in a direction parallel to the crack edges. The presence of the current is reflected in the form of the governing plate equation - a modified (orthotropic) version of the usual plate equation. A time-harmonic flexural wave is incident at arbitrary angle to the crack and undergoes scattering at the crack tip. The model problem is solved exactly using the Wiener-Hopf technique. The aim of this paper is to determine how the presence of the electric current affects both the generation of edge waves on the crack faces and the edge diffracted field. The Kirchhoff bending and twisting stress intensity factors are also studied.

This investigation differs from [1] in that Ambur et al. considered a crack of finite length and only the case of symmetric forcing; they formulated the problem as an integral equation and employed a numerical solution procedure. The present model problem is simpler in form, which allows an exact solution and thus enables a more detailed understanding of the physics of the problem. Further, the focus of [1] was the effect of the current on the bending moment intensity factor (herein referred to as the Kirchhoff stress intensity factor, $\bar{\kappa}_{1}$ ) whereas here, as well as the bending and twisting moment stress intensity factors, the diffracted and edge wave fields are also examined.

It is convenient to decompose the problem into its symmetric and anti-symmetric component parts and, in section two, these sub-problems are formulated. In section three, the Wiener-Hopf procedure is used to obtain a closed form solution to both subproblems. The physical features of the Wiener-Hopf solution are discussed in section four and a range of numerical results are presented, whilst, in the final section, the findings are discussed and some conclusions drawn.

\section{Formulation of the boundary value problem}

An isotropic, elastic plate, of thickness $2 h$, Poisson's ratio $\nu$, Young's modulus $E$ and density $\rho$, is assumed to lie in the $\bar{x} \bar{y}$-plane of a Cartesian coordinate system.

\footnotetext{
${ }^{1}$ It has recently been pointed out, see [3], that an earlier work on stability of static plate deflections by Ishlinskii [4] presented eigenfrequencies and dispersion relations that are closely related to those of Konenkov.
} 
The plate carries a uniform, constant electric current of density $\left(I_{0}, 0,0\right)$, see figure 1. The dimensional in-vacuo time-harmonic flexural displacement of the plate, $\Re\left\{\bar{w}(\bar{x}, \bar{y}) e^{-i \omega \bar{t}}\right\}$, is governed by the modified plate equation derived in [1]. That is,

$$
D\left(\frac{\partial^{4}}{\partial \bar{x}^{4}}+2 \frac{\partial^{4}}{\partial \bar{x}^{2} \partial \bar{y}^{2}}+\frac{\partial^{4}}{\partial \bar{y}^{4}}\right) \bar{w}-2 \rho h \omega^{2} \bar{w}-J_{0}^{2}\left(\bar{w}+\frac{h^{2}}{3} \frac{\partial^{2} \bar{w}}{\partial \bar{x}^{2}}\right)=0
$$

where $\omega$ is the angular frequency of the steady-state flexural wave field, $D=2 E h^{3} /(3(1-$ $\left.\nu^{2}\right)$ ) is the plate bending stiffness and $J_{0}=\sqrt{2 h \mu_{r} \mu_{0}} I_{0}$ in which $\mu_{0}, \mu_{r}$ are respectively the magnetic permeability in vacuo and the relative magnetic permeability of the plate. The reader is referred to [1] and [11] for full details of the derivation of this and related equations; note, however, that herein the unscaled (scaled) current density are denoted by as $I_{0}\left(J_{0}\right)$, whereas in Ambur et al. it is written as $J_{0}\left(\tilde{J}_{0}\right)$. It is convenient to non-dimensionalise variables with respect to time and length scales $\omega^{-1}$ and $k^{-1}$ respectively, where

$$
k=\left(\frac{2 \rho h \omega^{2}+J_{0}^{2}}{D}\right)^{1 / 4}
$$

and thus $k \bar{w}=w$ etc. Then,

$$
\frac{\partial^{4} w}{\partial x^{4}}+2 \frac{\partial^{4} w}{\partial x^{2} \partial y^{2}}+\frac{\partial^{4} w}{\partial y^{4}}-w-\epsilon^{2} \frac{\partial^{2} w}{\partial x^{2}}=0
$$

where

$$
\epsilon^{2}=\frac{J_{0}^{2} h^{2}}{3 D k^{2}}
$$

This somewhat unusual scaling (2.2) ensures that the dimensionless governing equation contains the single parameter $\epsilon$, which has different limiting behaviours according to whether $\omega \rightarrow \infty$ or $J_{0} \rightarrow \infty$.

Forcing is introduced in the form of a plane flexural wave, of unit amplitude, incident at angle $\theta$ to the $x$-axis (the crack is chosen to lie along the negative $x$-axis). Thus, the incident wave has the form

$$
w_{\text {inc }}(x, y)=\exp \{-i \lambda(x \cos \theta+y \sin \theta)\}
$$

where, on substituting (2.5) into (2.3), the wavenumber $\lambda$ is given by

$$
\lambda(\theta)=\sqrt{-\frac{\epsilon^{2} \cos ^{2} \theta}{2}+\sqrt{\frac{\epsilon^{4} \cos ^{4} \theta}{4}+1}} .
$$

Note that, here and henceforth, if $\lambda$ is written without an explicit argument it will be taken to be this function of the incident angle $\theta$. At a traction free crack the bending moment $M_{y}$ and the effective Kirchhoff shear force ${ }^{2} V_{y}=Q_{y}+M_{y x, x}$ must both vanish. Thus,

$$
\begin{aligned}
\frac{\partial^{2} w}{\partial y^{2}}+\nu \frac{\partial^{2} w}{\partial x^{2}} & =0 \\
\frac{\partial^{3} w}{\partial y^{3}}+(2-\nu) \frac{\partial^{3} w}{\partial y \partial x^{2}} & =0
\end{aligned}
$$




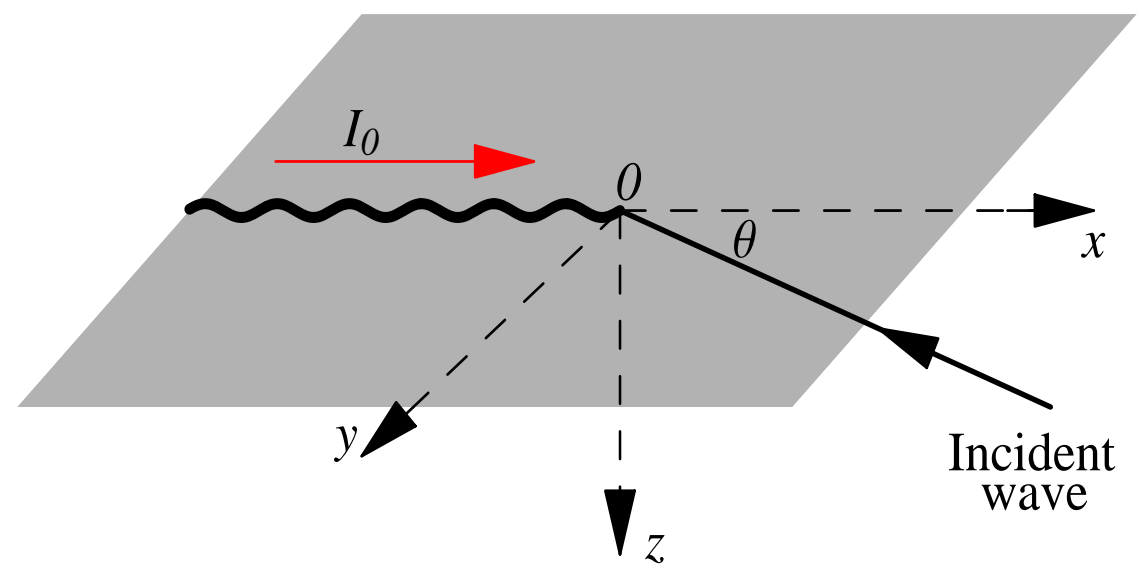

Figure 1: Geometry of the plate showing the location of the crack, the incident wave and direction of the current.

where the quantity $w(x, y)$ denotes the total plate displacement, that is, the incident wave $w_{\text {inc }}(x, y)$, and the scattered field $w_{s}(x, y)$.

For ease of exposition the cases of symmetric and anti-symmetric displacements are considered separately. Thus, the plate displacement may be expressed as

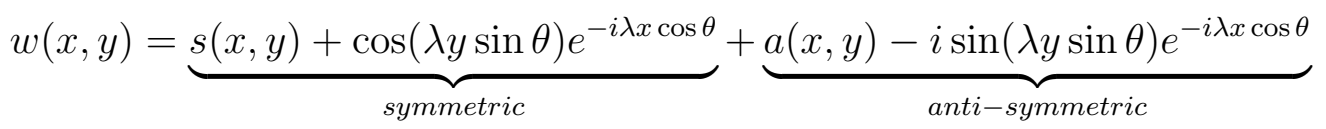

where $s(x, y)$ and $a(x, y)$ denote the symmetric and anti-symmetric components of the scattered field respectively. The boundary value problems for the symmetric and anti-symmetric scattered fields can now be formulated.

\subsection{Symmetric case}

The symmetric component of the displacement satisfies the modified plate equation (2.3). Since the displacement is symmetric about the line $y=0$, its odd derivatives in $y$ must be zero along $y=0, x>0$ (where the plate is continuous). This implies that (2.8) holds for $x>0$ as well as along the crack. Thus, expressed in terms of $s(x, y)$, the appropriate boundary conditions for symmetric displacement are

$$
\begin{aligned}
\frac{\partial s}{\partial y} & =0, \quad y=0, x>0, \\
\frac{\partial^{3} s}{\partial y^{3}}+(2-\nu) \frac{\partial^{3} s}{\partial x^{2} \partial y} & =0, \quad y=0,-\infty<x<\infty, \\
\frac{\partial^{2} s}{\partial y^{2}}+\nu \frac{\partial^{2} s}{\partial x^{2}} & =\lambda^{2} f^{s}(\theta) e^{-i \lambda x \cos \theta}, \quad y=0, x<0,
\end{aligned}
$$

\footnotetext{
${ }^{2}$ For a derivation of this condition see, for example, the book by Graff [12]; note that the notation used herein differs slightly from that in [1] but is consistent with [12].
} 
where $f^{s}(\theta)=\sin ^{2} \theta+\nu \cos ^{2} \theta$.

\subsection{Anti-symmetric case}

The anti-symmetric component of the displacement also satisfies the modified plate equation (2.3). In this case, however, displacement (and even derivatives in $y$ ) must be zero along $y=0, x>0$. This implies that (2.7) holds for $x>0$ as well as along the crack. Thus, expressed in terms of $a(x, y)$, the appropriate boundary conditions for anti-symmetric displacement are

$$
\begin{aligned}
a & =0, \quad y=0, x>0, \\
\frac{\partial^{2} a}{\partial y^{2}}+\nu \frac{\partial^{2} a}{\partial x^{2}} & =0, \quad y=0,-\infty<x<\infty, \\
\frac{\partial^{3} a}{\partial y^{3}}+(2-\nu) \frac{\partial^{3} a}{\partial x^{2} \partial y} & =-i \lambda^{3} f^{a}(\theta) e^{-i \lambda x \cos \theta}, y=0, x<0,
\end{aligned}
$$

where $f^{a}(\theta)=\sin ^{3} \theta+(2-\nu) \cos ^{2} \theta \sin \theta$.

\section{The Wiener-Hopf Procedure}

Both the symmetric and anti-symmetric cases described above constitute typical Wiener-Hopf problems. For readers who are unfamiliar with the Wiener-Hopf technique, reference [13] provides a useful history whilst [14] is the definitive introductory text. The procedure commences via Fourier transformation in the $x$-direction, where the transform of the total scattered displacement is here defined as

$$
\begin{aligned}
W(\alpha, y)=\int_{-\infty}^{\infty} w_{s}(x, y) e^{i \alpha x} d x & =\int_{-\infty}^{\infty} s(x, y) e^{i \alpha x} d x+\int_{-\infty}^{\infty} a(x, y) e^{i \alpha x} d x \\
& =S(\alpha, y)+A(\alpha, y) .
\end{aligned}
$$

The inverse Fourier transform is similarly defined as

$$
w_{s}(x, y)=\frac{1}{2 \pi} \int_{-\infty}^{\infty} W(\alpha, y) e^{-i \alpha x} d \alpha
$$

where the path of integration in the $\alpha$-plane lies along the real line indented above (below) any poles on the negative (positive) real axis. Note that the pole due to the incident wave term, which may lie on either the positive or negative side of the real line, is always traversed by indentation of the contour below this singularity. The reasons for the choice of indentation will become clear later.

It is necessary to also define half range Fourier transforms, that is,

$$
\begin{aligned}
W_{+}(\alpha, y) & =\int_{0}^{\infty} w_{s}(x, y) e^{i \alpha x} d x \\
& =S_{+}(\alpha, y)+A_{+}(\alpha, y)
\end{aligned}
$$

and

$$
\begin{aligned}
W_{-}(\alpha, y) & =\int_{-\infty}^{0} w_{s}(x, y) e^{i \alpha x} d x \\
& =S_{-}(\alpha, y)+A_{-}(\alpha, y) .
\end{aligned}
$$


Here and henceforth the subscript + and - indicate functions which are analytic in overlapping upper and lower halves of the complex $\alpha$-plane, respectively. Note that the integration path of (3.17) lies in the overlap region.

On taking the full-range Fourier transform of (2.3), it is easily seen that

$$
W^{\prime \prime \prime \prime}-2 \alpha^{2} W^{\prime \prime}+\left(\alpha^{4}-1+\epsilon^{2} \alpha^{2}\right) W=0,
$$

where prime indicates differentiation with respect to $y$. The solution to this ordinary differential equation that decays or has outgoing wave behaviour as $y \rightarrow \infty$ is

$$
W(\alpha, y)=C(\alpha) e^{-\gamma_{1} y}+D(\alpha) e^{-\gamma_{2} y}
$$

where $\gamma_{1}=\sqrt{\alpha^{2}+\gamma_{3}}, \gamma_{2}=\sqrt{\alpha^{2}-\gamma_{3}}$ and $\gamma_{3}=\sqrt{1-\epsilon^{2} \alpha^{2}}=\sqrt{\lambda^{4}+\epsilon^{2} \lambda^{2} \cos ^{2} \theta-\epsilon^{2} \alpha^{2}}$. The branch-cut locations and chosen Riemann sheets of these functions, which yield the required property for $W(\alpha, y)$, will be described in Section four. Note that, due to the fact that the full problem is to be solved in terms of its symmetric and antisymmetric component parts, it is sufficient to consider only the half-plane $y \geq 0$. The functions $C(\alpha)$ and $D(\alpha)$ are now determined using the boundary conditions and will take different forms for the symmetric and anti-symmetric cases.

\subsection{Symmetric case}

On applying the full range Fourier transform to (2.11), it is found that

$$
\alpha^{2}(2-\nu) S^{\prime}-S^{\prime \prime \prime}=0, \quad y=0
$$

and it follows, on substituting (3.21) into (3.22), that

$$
D^{s}(\alpha)=-\frac{\gamma_{1}\left[\alpha^{2}(\nu-1)+\gamma_{3}\right]}{\gamma_{2}\left[\alpha^{2}(\nu-1)-\gamma_{3}\right]} C^{s}(\alpha)
$$

where the superscript $s$ denotes that these functions are specific to the symmetric problem. Thus, the symmetric part of equation (3.21) now becomes

$$
S(\alpha, y)=-\frac{\gamma_{1} C^{s}(\alpha)}{\left[\alpha^{2}(\nu-1)-\gamma_{3}\right]}\left\{\frac{\gamma_{3}+(1-\nu) \alpha^{2}}{\gamma_{1}} e^{-\gamma_{1} y}+\frac{\gamma_{3}-(1-\nu) \alpha^{2}}{\gamma_{2}} e^{-\gamma_{2} y}\right\},
$$

which may be recast as

$$
S(\alpha, y)=\frac{E^{s}(\alpha)}{2 \gamma_{3}}\left\{\frac{\gamma_{3}+(1-\nu) \alpha^{2}}{\gamma_{1}} e^{-\gamma_{1} y}+\frac{\gamma_{3}-(1-\nu) \alpha^{2}}{\gamma_{2}} e^{-\gamma_{2} y}\right\}
$$

where the quantity $E^{s}(\alpha)$ is unknown and is determined using the remaining boundary conditions and the Wiener-Hopf procedure.

On taking the appropriate half range transforms of boundary conditions (2.10) and (2.12), it is found that

$$
S_{+}^{\prime}(\alpha, 0)=0
$$

and

$$
S_{-}^{\prime \prime}(\alpha, 0)-\alpha^{2} \nu S_{-}(\alpha, 0)+\nu b^{s}-i \nu c^{s} \alpha=\frac{-i \lambda^{2} f^{s}(\theta)}{(\alpha-\lambda \cos \theta)_{-}}
$$


where $c^{s}=s\left(0^{-}, 0\right), b^{s}=s_{x}\left(0^{-}, 0\right)$, and the - subscript on the right hand term indicates that the incident wave pole lies above the inverse contour, i.e. in the upper half-plane.

On differentiating (3.25) with respect to $y$, setting $y=0$ and using (3.26), it is found that

$$
S_{-}^{\prime}(\alpha, 0)=-E^{s}(\alpha) .
$$

Similarly, on using (3.25), it is found that

$$
\begin{aligned}
\Phi_{+}^{s}(\alpha) & +S_{-}^{\prime \prime}(\alpha, 0)-\alpha^{2} \nu S_{-}(\alpha, 0)= \\
& -\alpha^{2} \nu \frac{E^{s}(\alpha)}{2 \gamma_{3}}\left\{\frac{\left[\gamma_{3}+(1-\nu) \alpha^{2}\right]}{\gamma_{1}}+\frac{\left[\gamma_{3}-(1-\nu) \alpha^{2}\right]}{\gamma_{2}}\right\} \\
& +\frac{E^{s}(\alpha)}{2 \gamma_{3}}\left\{\gamma_{1}\left[\gamma_{3}+(1-\nu) \alpha^{2}\right]+\gamma_{2}\left[\gamma_{3}-(1-\nu) \alpha^{2}\right]\right\}
\end{aligned}
$$

where $\Phi_{+}^{s}(\alpha)=S_{+}^{\prime \prime}(\alpha, 0)-\alpha^{2} \nu S_{+}(\alpha, 0)$. This can be rearranged as

$$
\Phi_{+}^{s}(\alpha)+S_{-}^{\prime \prime}(\alpha, 0)-\alpha^{2} \nu S_{-}(\alpha, 0)=-S_{-}^{\prime}(\alpha, 0) K(\alpha)
$$

where

$$
K(\alpha)=\frac{1}{2 \gamma_{3}}\left\{\frac{\left[\gamma_{3}+(1-\nu) \alpha^{2}\right]^{2}}{\gamma_{1}}-\frac{\left[\gamma_{3}-(1-\nu) \alpha^{2}\right]^{2}}{\gamma_{2}}\right\}
$$

The Wiener-Hopf equation is now obtained by using (3.27):

$$
\Phi_{+}^{s}(\alpha)-\frac{i \lambda^{2} f^{s}(\theta)}{(\alpha-\lambda \cos \theta)_{-}}-\nu b^{s}+i \nu c^{s} \alpha=-S_{-}^{\prime}(\alpha, 0) K(\alpha) .
$$

In the usual manner, a product factorisation (see the Appendix for details) may be uniquely defined as

$$
K(\alpha)=K_{+}(\alpha) K_{-}(\alpha), \quad K_{-}(-\alpha)=K_{+}(\alpha),
$$

where $K_{ \pm}(\alpha)$ are analytic and zero free in their indicated half-planes. Then, on dividing (3.32) through by $K_{+}(\alpha)$ and performing a sum-split on the forcing term, the Wiener-Hopf equation can be rearranged as

$$
\begin{aligned}
\frac{\Phi_{+}^{s}(\alpha)}{K_{+}(\alpha)} & +\frac{i \nu c^{s} \alpha-\nu b^{s}}{K_{+}(\alpha)}-\frac{i \lambda^{2} f^{s}(\theta)}{\alpha-\lambda \cos \theta}\left\{\frac{1}{K_{+}(\alpha)}-\frac{1}{K_{+}(\lambda \cos \theta)}\right\} \\
& =F^{s}(\alpha)=\frac{i \lambda^{2} f^{s}(\theta)}{K_{+}(\lambda \cos \theta)(\alpha-\lambda \cos \theta)_{-}}-S^{\prime}{ }_{-}(\alpha, 0) K_{-}(\alpha) .
\end{aligned}
$$

This has been organised so that the left hand side is analytic in the upper half-plane whilst the right hand side is analytic in the overlapping lower half-plane. Thus, both sides offer an analytic continuation into the whole of the complex $\alpha$ plane and so must equate to an entire function, $F^{s}(\alpha)$ say. It follows that

$$
S^{\prime}{ }_{-}(\alpha, 0)=\frac{i \lambda^{2} f^{s}(\theta)}{K_{+}(\lambda \cos \theta) K_{-}(\alpha)(\alpha-\lambda \cos \theta)_{-}}-\frac{F^{s}(\alpha)}{K_{-}(\alpha)}
$$

and on using (3.35) and (3.26) it is found that

$$
\frac{\partial s}{\partial y}(x, 0)=\frac{1}{2 \pi} \int_{-\infty}^{\infty}\left\{\frac{i \lambda^{2} f^{s}(\theta)}{K_{+}(\lambda \cos \theta) K_{-}(\alpha)(\alpha-\lambda \cos \theta)_{-}}-\frac{F^{s}(\alpha)}{K_{-}(\alpha)}\right\} e^{-i \alpha x} d \alpha .
$$


Clearly, this is zero for $x>0$. Further, since the integral must exist for all $x$ and $K_{-}(\alpha)=O\left(\alpha^{1 / 2}\right)$ as $|\alpha| \rightarrow \infty$, it is straightforward to show that $F^{s}(\alpha)=0$. It follows, from (3.28) and (3.25) that the symmetric component of displacement is given by

$$
\begin{aligned}
s(x, y)= & \frac{-1}{2 \pi} \int_{-\infty}^{\infty} \frac{i \lambda^{2} f^{s}(\theta)}{K_{+}(\lambda \cos \theta) K_{-}(\alpha)(\alpha-\lambda \cos \theta)_{-}} \\
& \times\left\{\frac{\gamma_{3}+(1-\nu) \alpha^{2}}{\gamma_{1}} e^{-\gamma_{1} y}+\frac{\gamma_{3}-(1-\nu) \alpha^{2}}{\gamma_{2}} e^{-\gamma_{2} y}\right\} \frac{e^{-i \alpha x}}{2 \gamma_{3}} d \alpha .
\end{aligned}
$$

\subsection{Anti-symmetric case}

The Wiener-Hopf procedure follows in an analogous manner. Equivalent to (3.25) and (3.28), it is found from (2.13) and (2.14) that

$$
A(\alpha, y)=\frac{A_{-}(\alpha, 0)}{2 \gamma_{3}}\left\{\left[\gamma_{3}-(1-\nu) \alpha^{2}\right] e^{-\gamma_{1} y}+\left[\gamma_{3}+(1-\nu) \alpha^{2}\right] e^{-\gamma_{2} y}\right\} .
$$

On using the remaining boundary condition (2.15) the Wiener-Hopf equation is formulated as

$$
\Phi_{+}^{a}(\alpha)-\frac{\lambda^{3} f^{a}(\theta)}{(\alpha-\lambda \cos \theta)_{-}}+(2-\nu)\left(i \alpha c^{a}-b^{a}\right)=A_{-}(\alpha, 0) K^{a}(\alpha),
$$

where $\Phi_{+}^{a}(\alpha)=A_{+}^{\prime \prime \prime}(\alpha, 0)-\alpha^{2}(2-\nu) A_{+}^{\prime}(\alpha, 0), c^{a}=a_{y}\left(0^{+}, 0\right), b^{a}=a_{x y}\left(0^{+}, 0\right)$ and

$$
K^{a}(\alpha)=\gamma_{1} \gamma_{2} K(\alpha)
$$

A product factorisation for $K^{a}(\alpha)$ is defined in the same manner as (3.33). This enables the Wiener-Hopf procedure to be performed, and it follows that

$$
A_{-}(\alpha, 0)=\frac{F^{a}(\alpha)}{K_{-}^{a}(\alpha)}-\frac{\lambda^{3} f^{a}(\theta)}{K_{+}^{a}(\lambda \cos \theta) K_{-}^{a}(\alpha)(\alpha-\lambda \cos \theta)_{-}},
$$

where $F^{a}(\alpha)$ is an (as yet unknown) entire function. On considering the behaviour of Kirchhoff's shear force as $x \rightarrow 0^{+}$, and ensuring finite energy density at the crack tip, it is found that $F^{a}(\alpha)=0$. Thus, the exact solution to the anti-symmetric problem is

$$
\begin{aligned}
a(x, y)= & \frac{-1}{2 \pi} \int_{-\infty}^{\infty} \frac{\lambda^{3} f^{a}(\theta)}{K_{+}^{a}(\lambda \cos \theta) K_{-}^{a}(\alpha)(\alpha-\lambda \cos \theta)_{-}} \\
& \times\left\{\left[\gamma_{3}-(1-\nu) \alpha^{2}\right] e^{-\gamma_{1} y}+\left[\gamma_{3}+(1-\nu) \alpha^{2}\right] e^{-\gamma_{2} y}\right\} \frac{e^{-i \alpha x}}{2 \gamma_{3}} d \alpha
\end{aligned}
$$

\section{Analysis of the solution}

For both the symmetric and anti-symmetric sub-problems considered above, the symmetric kernel $K(\alpha)$ is fundamental to the underlying physics. Recall that

$$
K(\alpha)=\frac{1}{2 \gamma_{3}}\left\{\frac{\left[\gamma_{3}+(1-\nu) \alpha^{2}\right]^{2}}{\gamma_{1}}-\frac{\left[\gamma_{3}-(1-\nu) \alpha^{2}\right]^{2}}{\gamma_{2}}\right\}
$$


where $\gamma_{1}=\sqrt{\alpha^{2}+\gamma_{3}}, \gamma_{2}=\sqrt{\alpha^{2}-\gamma_{3}}$ and $\gamma_{3}=\sqrt{1-\epsilon^{2} \alpha^{2}}$, and hence

$$
K(\alpha) \sim \frac{|\alpha|}{2}(3+\nu)(1-\nu) \quad \text { as } \quad|\alpha| \rightarrow \infty .
$$

Note that the branch-cut functions are related via

$$
\gamma_{1}^{2}+\gamma_{2}^{2}=2 \alpha^{2} \quad \text { and } \quad \gamma_{1}^{2}-\gamma_{2}^{2}=2 \gamma_{3}
$$

in which $\gamma_{1}$ and $\gamma_{2}$ have branch-points at $\alpha_{1}$ and $\alpha_{2}$ respectively, where

$$
\alpha_{1}=i \sqrt{\frac{\sqrt{\epsilon^{4}+4}+\epsilon^{2}}{2}} \sim \begin{cases}i+\frac{i \epsilon^{2}}{4}, & \epsilon \rightarrow 0, \\ i \epsilon+\frac{i}{2 \epsilon^{3}}, & \epsilon \rightarrow \infty\end{cases}
$$

and

$$
\alpha_{2}=\sqrt{\frac{\sqrt{\epsilon^{4}+4}-\epsilon^{2}}{2}} \sim\left\{\begin{array}{l}
1-\frac{\epsilon^{2}}{4}, \quad \epsilon \rightarrow 0, \\
\frac{1}{\epsilon}-\frac{1}{2 \epsilon^{5}}, \quad \epsilon \rightarrow \infty .
\end{array}\right.
$$

The branch-cuts from $\pm \alpha_{i}, i=1,2$, and those at $\alpha= \pm 1 / \epsilon$, are taken to infinity respectively in the upper/lower half planes. The sheet of the Riemann surface is chosen which, on the real line between the respective branch-points, yields either positive real or purely negative imaginary values for the three square-root functions. This means that the kernel has zeros at $\pm \alpha_{e d}$ and $\pm \alpha_{e v}$ which correspond to an edge wave and an evanescent mode. These roots can be located analytically; it is found that

$$
\alpha_{e d}=\left(\frac{\sqrt{p^{4} \epsilon^{4}+4 p^{2}\left(p^{2}-4\right)}-p^{2} \epsilon^{2}}{2\left(p^{2}-4\right)}\right)^{1 / 2} \sim \begin{cases}\frac{p^{1 / 2}}{\left(p^{2}-4\right)^{1 / 4}}-\frac{p^{3 / 2} \epsilon^{2}}{4\left(p^{2}-4\right)^{3 / 4}}, & \epsilon \rightarrow 0, \\ \frac{1}{\epsilon}-\frac{\left(p^{2}-4\right)}{2 p^{2} \epsilon^{5}}, & \epsilon \rightarrow \infty,\end{cases}
$$

and

$$
\alpha_{e v}=i\left(\frac{\sqrt{p^{4} \epsilon^{4}+4 p^{2}\left(p^{2}-4\right)}+p^{2} \epsilon^{2}}{2\left(p^{2}-4\right)}\right)^{1 / 2} \sim \begin{cases}\frac{i p^{1 / 2}}{\left(p^{2}-4\right)^{1 / 4}}+\frac{i p^{3 / 2} \epsilon^{2}}{4\left(p^{2}-4\right)^{3 / 4}}, & \epsilon \rightarrow 0 \\ \frac{i p \epsilon}{\sqrt{p^{2}-4}}+\frac{i \sqrt{p^{2}-4}}{2 p \epsilon^{3}}, & \epsilon \rightarrow \infty\end{cases}
$$

where $p=2(1-\nu+\sqrt{1+2 \nu(\nu-1)}) / \nu^{2}$. It is straightforward to show that the quantity $\left(p^{2}-4\right) / p^{2}$ tends to unity as $\nu \rightarrow 0$ and from there decreases monotonically as $\nu \rightarrow 1 / 2$. Thus, after a little algebra, it follows that $0<\alpha_{2}<\alpha_{e d}<1 / \epsilon$ as $\epsilon \rightarrow \infty$ for $0<\nu<1 / 2$. In fact, it can be verified numerically that the edge wave pole lies between the branch-point $\alpha_{2}$ and the branch-point of $\gamma_{3}, 1 / \epsilon$, for all values of $\epsilon$.

\subsection{Steepest descent analysis}

The exact solution of the boundary value problem was obtained in Section 3. The form presented in (3.37) and (3.41) is not particularly helpful for evaluation purposes, so the standard approach is to obtain the diffracted far-field behaviour together with any propagating wave terms. To achieve this end the integration contour is deformed into the steepest descent path(s). It can be seen that the combined scattered field $w_{s}(x, y)=s(x, y)+a(x, y)$ is composed of an integral with two exponential terms in the integrand: $e^{-\gamma_{1} y-i \alpha x}$ and $e^{-\gamma_{2} y-i \alpha x}$. The usual approach is thus to separate $w_{s}$ 
into two integrals before deforming each into its own steepest descent path. Doing this, however, introduces the branch-cuts in $\gamma_{3}$, whereas the combined integral is free of these singularities. It is preferable, therefore, to keep the integrals together and deform into the steepest descent curve for the $\gamma_{2}$ term, on which it can be shown, in a similar fashion to that discussed in detail in [15] and [16], that the contribution from the $\gamma_{1}$ exponent is always exponentially smaller than that from the $\gamma_{2}$ exponent.

On introducing the change of variables

$$
x=r \cos \psi, \quad y=r \sin \psi,
$$

the saddle point is deduced from the vanishing of the derivative of the phase factor $\chi(\alpha)$, where

$$
r \chi(\alpha)=\gamma_{2}(\alpha) y+i \alpha x=r\left(\gamma_{2}(\alpha) \sin \psi+i \alpha \cos \psi\right)
$$

This yields

$$
\left.\frac{\alpha\left(2 \gamma_{3}(\alpha)+\epsilon^{2}\right)}{2 \gamma_{2}(\alpha) \gamma_{3}(\alpha)}\right|_{\alpha=\alpha_{s}}=-i \cot \psi
$$

at the saddle point $\alpha_{s}$, which, following [16] we choose to write as

$$
\alpha_{s}=-\lambda(\beta) \cos \beta
$$

with $\lambda(\beta)$ given in (2.6). Substituting (4.51) into (4.50) yields a relationship between $\psi$ and $\beta$, namely

$$
\cot \beta\left(1+\frac{\epsilon^{2}}{2 \lambda^{2}(\beta)}\right)=\cot \psi,
$$

or, writing

$$
Q(\beta)=\frac{2 \lambda^{2}(\beta)}{2 \lambda^{2}(\beta)+\epsilon^{2}},
$$

then

$$
\cos \psi=\frac{\cos \beta}{\sqrt{\cos ^{2} \beta+Q^{2}(\beta) \sin ^{2} \beta}}, \quad \sin \psi=\frac{Q(\beta) \sin \beta}{\sqrt{\cos ^{2} \beta+Q^{2}(\beta) \sin ^{2} \beta}} .
$$

Note that $\beta$ is a transformed angle, which takes account of the anisotropy of the governing plate equation. The saddle moves from $-\alpha_{2}$ when $\beta=0$ to $+\alpha_{2}$ when $\beta=\pi$, and the shadow boundary is located at

$$
\beta=\pi-\theta, \quad 0 \leq \beta \leq \pi,
$$

(and at $\beta=\theta-\pi$ in the region $y<0)$ not at the physical angle $\psi=\pi-\theta$ (or $\theta-\pi)$.

Near the saddle point the phase function behaves as

$$
\chi(\alpha) \sim \chi\left(\alpha_{s}\right)+\frac{1}{2 !}\left(\alpha-\alpha_{s}\right)^{2} \chi^{\prime \prime}\left(\alpha_{s}\right),
$$

where

$$
\chi\left(\alpha_{s}\right)=-i \lambda(\beta) \frac{\cos ^{2}(\beta)+Q(\beta) \sin ^{2} \beta}{\sqrt{\cos ^{2} \beta+Q^{2}(\beta) \sin ^{2} \beta}}=-i \lambda(\beta) \cos (\beta-\psi),
$$

and so the steepest descent path is defined as the contour on which

$$
\Im(\chi(\alpha))=\Im\left(\chi\left(\alpha_{s}\right)\right), \quad \Re(\chi(\alpha)) \geq \Re\left(\chi\left(\alpha_{s}\right)\right) .
$$


The details of the contour are similar to those found in other articles (e.g. [16] and [17]) and so are omitted here for brevity. Once on the steepest descent path, a uniform result for the saddle point contribution is obtained in a straightforward manner. This gives, after a little algebra,

$$
w_{s} \sim F\left(\alpha_{s}\right) e^{-r \chi\left(\alpha_{s}\right)} e^{-i r\left(\lambda \cos \theta-\alpha_{s}\right)^{2}\left|\chi^{\prime \prime}\left(\alpha_{s}\right)\right| / 2} \operatorname{erfc}\left[\sqrt{\frac{r}{2}} e^{-i \pi / 4}\left(\lambda \cos \theta-\alpha_{s}\right)\left|\chi^{\prime \prime}\left(\alpha_{s}\right)\right|^{1 / 2}\right]
$$

where

$$
F\left(\alpha_{s}\right)=\frac{\lambda^{2} f^{s}(\theta)\left(\gamma_{3}\left(\alpha_{s}\right)-(1-\nu) \alpha_{s}^{2}\right)}{4 \gamma_{2}\left(\alpha_{s}\right) \gamma_{3}\left(\alpha_{s}\right) K_{-}\left(\alpha_{s}\right) K_{+}(\lambda \cos \theta)}-\frac{i \lambda^{3} f^{a}(\theta)\left(\gamma_{3}\left(\alpha_{s}\right)+(1-\nu) \alpha_{s}^{2}\right)}{4 \gamma_{3}\left(\alpha_{s}\right) K_{-}^{a}\left(\alpha_{s}\right) K_{+}^{a}(\lambda \cos \theta)} .
$$

The reader is reminded that the appearance of $\lambda$ without an argument should be read as $\lambda(\theta)$.

The complementary error function, erfc $[z]$, is a decaying function as $\Im z<0$, but is equal to $2-\operatorname{erfc}[-z]$ for $\Im(z)>0$; the latter has the contribution of the specular reflected wave term contained within it when the shadow boundary has been crossed. From this, and the asymptotic result

$$
e^{-i z^{2}} \operatorname{erfc}\left(z e^{-i \pi / 4}\right) \sim \frac{e^{i \pi / 4}}{\sqrt{\pi} z}, \quad|z| \rightarrow \infty, \Re z>0,
$$

we deduce (for $\lambda \cos \theta>\alpha_{s}$ )

$$
w_{s} \sim \sqrt{\frac{2}{\pi r}} \frac{F\left(\alpha_{s}\right) e^{-r \chi\left(\alpha_{s}\right)} e^{i \pi / 4}}{\left(\lambda \cos \theta-\alpha_{s}\right)\left|\chi^{\prime \prime}\left(\alpha_{s}\right)\right|^{1 / 2}}, \quad \beta<\pi-\theta,
$$

and (when $\lambda \cos \theta<\alpha_{s}$ )

$$
\begin{aligned}
w_{s} & \sim 2 F\left(\alpha_{s}\right) e^{i \lambda(x \cos \theta+y \sin \theta)} \\
& -\sqrt{\frac{2}{\pi r}} \frac{F\left(\alpha_{s}\right) e^{-r \chi\left(\alpha_{s}\right)} e^{i \pi / 4}}{\left(\alpha_{s}-\lambda \cos \theta\right)\left|\chi^{\prime \prime}\left(\alpha_{s}\right)\right|^{1 / 2}}, \quad \beta>\pi-\theta,
\end{aligned}
$$

as $r \rightarrow \infty$ as long as $\beta$ is not too close to $\pi-\theta$.

It can be shown that deformation of the Fourier integral contour onto the steepest descent path crosses the edge wave pole $\alpha_{e d}$ for observation angle $\psi$ close to $\pi$, i.e. near the crack edge. Further, it is found that the deformation never crosses the evanescent pole at $\alpha_{e v}$. Thus, the contribution from the edge wave must be added to the far-field diffracted and specular terms $(4.62,4.63)$ to obtain the total scattered field.

\subsection{The edge wave}

Of primary interest is the effect on the edge wave of varying the scaled current density $J_{0}$. There are two features to be investigated: the phase speed and the amplitude. For the numerical results presented herein an aluminium plate of thickness $0.004 \mathrm{~m}$ is considered. Thus, typical values are chosen as: $h=0.002 \mathrm{~m}, \rho=2.743 \times 10^{3} \mathrm{~kg} \mathrm{~m}^{-3}$, $E=6.9 \times 10^{10} \mathrm{Nm}^{-2}, \mu_{r}=1$ and $\nu=0.33$. For consistency with Ambur et al. [1], the maximum current density $I_{0}$ will be taken as $2 \times 10^{9} \mathrm{~A} \mathrm{~m}^{-2}$, although such high values will almost certainly be unfeasible for most engineering applications. 
Asymptotic expressions for the phase speed $c$ as the radian frequency $\omega \rightarrow \infty$ or current density $J_{0} \rightarrow \infty$ are readily available since these limits correspond respectively to $\epsilon \rightarrow 0$ and $\epsilon \rightarrow \infty$. Thus,

$$
c=\frac{\omega}{k \alpha_{e d}} \sim\left(\frac{\omega^{2}\left(p^{2}-4\right) D}{2 p^{2} \rho h}\right)^{1 / 4}, \quad \omega \rightarrow \infty
$$

and

$$
c=\frac{\omega}{k \alpha_{e d}} \sim \frac{\omega h}{\sqrt{3}}, \quad J_{0} \rightarrow \infty .
$$

These two limiting behaviours are demonstrated in figure 2. Note that the constant asymptotic limit (4.65) is clearly visible in figure 2(b), but in order to attain this limit the values of $J_{0}$ exceed those that are physically realistic in this context. Also of interest is the ratio of the phase speed of edge waves to the phase speed of flexural waves, which can be determined exactly:

$$
\frac{c}{\left.c_{\text {inc }}\right|_{\theta=0}}=\sqrt{\frac{p^{2}\left(\sqrt{\epsilon^{4}+4\left(p^{2}-4\right) / p^{2}}-\epsilon^{2}\right)}{\left(p^{2}-4\right)\left(\sqrt{\epsilon^{4}+4}-\epsilon^{2}\right)}} .
$$

This, as for the original Konenkov wave [2], shows remarkably little deviation from unity whatever the current.

Phase Speed

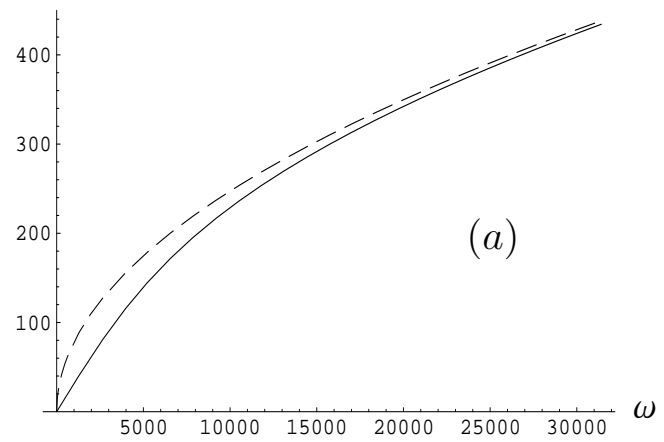

Phase Speed

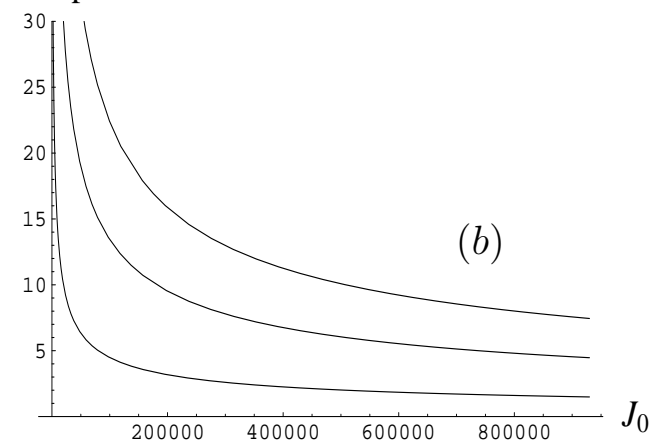

Figure 2: (a) Phase speed of the edge wave plotted against frequency for $J_{0}=0$ (dashed curve) and $J_{0}=20000 \mathrm{~kg}^{1 / 2} \mathrm{~s}^{-1} \mathrm{~m}^{-1}$, and (b) the phase speed against $J_{0}$ for frequencies of $50 \mathrm{~Hz}$ (lowest curve), $150 \mathrm{~Hz}$ and $250 \mathrm{~Hz}$ (uppermost curve).

The symmetric and anti-symmetric components of the edge wave amplitude are obtained from (3.37) and (3.41) by deforming the path of integration onto the steepest descent contour, and picking up a contribution from the residue $\alpha=\alpha_{e d}$ in the vicinity of the crack. On putting $y=0$ and using (4.43), it is found that the flexural edge wave term is

$$
w_{e d}=s_{e d}+a_{e d}
$$


where

$$
\begin{aligned}
s_{e d}= & \frac{-\lambda^{2} f^{s}(\theta) K_{+}\left(\alpha_{e d}\right)}{K^{\prime}\left(\alpha_{e d}\right)\left(\alpha_{e d}-\lambda \cos \theta\right)_{-} K_{+}(\lambda \cos \theta)} \\
& \times\left\{\frac{\gamma_{1}\left(\alpha_{e d}\right) \gamma_{2}\left(\alpha_{e d}\right)+\alpha_{e d}^{2} \nu}{\sqrt{2} \gamma_{1}\left(\alpha_{e d}\right) \gamma_{2}\left(\alpha_{e d}\right) \sqrt{\alpha_{e d}^{2}+\gamma_{1}\left(\alpha_{e d}\right) \gamma_{2}\left(\alpha_{e d}\right)}}\right\}
\end{aligned}
$$

and

$$
a_{e d}=\frac{-i \lambda^{3} f^{a}(\theta) K_{+}^{a}\left(\alpha_{e d}\right)}{K_{+}^{a}(\lambda \cos \theta)\left(\alpha_{e d}-\lambda \cos \theta\right)_{-} K^{a^{\prime}}\left(\alpha_{e d}\right)} .
$$

Figure 3(a) shows the absolute value of the dimensional amplitude of the edge wave $\left(\left|\bar{s}_{e d}+\bar{a}_{e d}\right|=\left|s_{e d}+a_{e d}\right| / k\right)$ against angular frequency and $J_{0}$ for $\theta=\pi / 1000$. It is clear that the amplitude decreases monotonically as either the frequency or current is increased. It is important to note that most of the dependence on frequency arises due to the dependence on $k$. Thus, the non-dimensional equivalent of this graph is nearly frequency independent, especially as $J_{0} \rightarrow \infty$. Figure 3(b) shows the nondimensional amplitude of the edge wave, at a frequency of $500 \mathrm{~Hz}$ and $J_{0}=100 \mathrm{~kg}^{1 / 2}$ $\mathrm{s}^{-1} \mathrm{~m}^{-1}$, plotted against $\theta$. The edge wave is excited principally for incoming waves in the first and fourth quadrants $(|\theta|<\pi / 2)$, with little energy conversion into the edge wave in the back-scattered direction. This picture is typical for all values of $J_{0}$ and $\omega$.
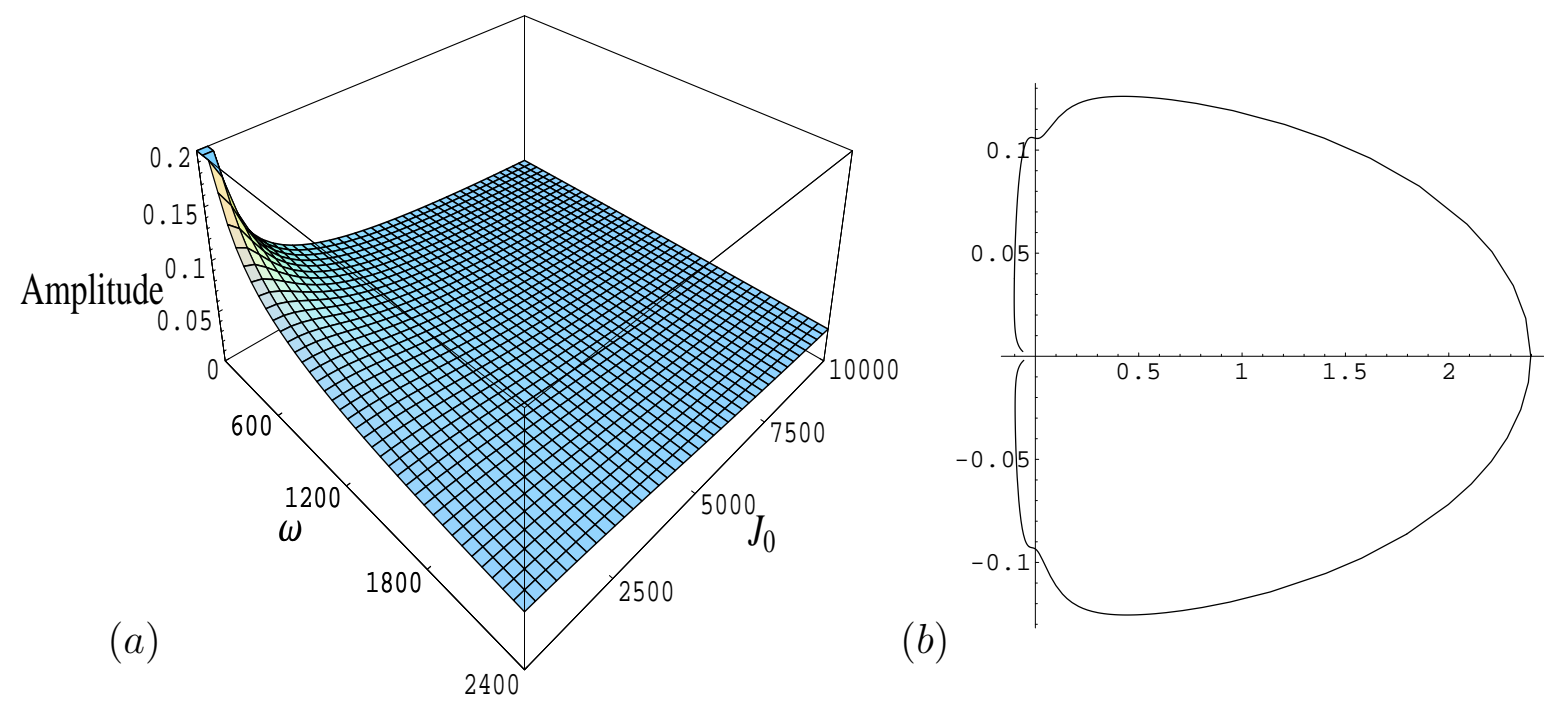

Figure 3: (a) Dimensional amplitude of the edge wave plotted against $\omega$ and $J_{0}$ and (b) the non-dimensional edge wave amplitude as a polar plot against angle of incidence at $500 \mathrm{~Hz}$ and $J_{0}=100 \mathrm{~kg}^{1 / 2} \mathrm{~s}^{-1} \mathrm{~m}^{-1}$. 


\subsection{Kirchhoff stress intensity factors}

Of significant engineering interest are the crack tip stress fields generated in a loaded, cracked plate. These can be modelled using 3-D elasticity [12] or a plate theory such as Reissner-Mindlin or Kirchhoff. Plate theories are not valid for thick plates or in the crack tip plastic zone, the latter deficiency being related to the stress field singularities located at the crack tip. Further, Kirchhoff theory cannot exactly satisfy the stress free conditions at the crack, whereas the more sophisticated Reissner-Mindlin theory (and other higher order models) can. Nevertheless, both plate theories yield stress fields that are valid outside the plastic crack tip and free edge zones. In particular, for $r>h$ where $r$ is the radial distance from the crack tip, Kirchhoff theory gives a good approximation to the three dimensional stress fields, see e.g. [19]. Additionally, Kirchhoff theory is easy to implement and provides the correct energy release rate ${ }^{3}$ [20]. Thus, the Kirchhoff stress intensity factors are viewed as a useful guide as to the strength of the stress or bending/twisting moment fields close to (though not at) the crack tip.

There is some variation in the definition of the stress intensity factors (cf. [21], [20]); however, the following definitions are used herein

$$
\bar{\kappa}_{1}=\lim _{x \rightarrow 0^{+}} \sqrt{2 \pi x} \bar{\sigma}_{y y}(x, 0, h) \text { and } \quad \bar{\kappa}_{2}=\lim _{x \rightarrow 0^{+}} \sqrt{2 \pi x} \bar{\sigma}_{x y}(x, 0, h),
$$

where, as in (2.1) the overbars indicate that these are dimensional quantities. The Kirchhoff stress intensity factors $\bar{\kappa}_{1}$ and $\bar{\kappa}_{2}$ are respectively the coefficients of the singularities in the stress fields just ahead of the crack for a bending mode and an anti-symmetric twisting-transverse shear mode. Ambur et al. [1] considered only symmetric forcing and thus only $\bar{\kappa}_{1}$ was relevant to their study.

Kirchhoff theory gives the following expressions for the shear stresses:

$$
\bar{\sigma}_{y y}(x, y, h)=-\frac{E h}{1-\nu^{2}}\left(\frac{\partial^{2} \bar{s}}{\partial \bar{y}^{2}}+\nu \frac{\partial^{2} \bar{s}}{\partial \bar{x}^{2}}\right)=-\frac{E k h}{1-\nu^{2}}\left(\frac{\partial^{2} s}{\partial y^{2}}+\nu \frac{\partial^{2} s}{\partial x^{2}}\right)
$$

and

$$
\bar{\sigma}_{x y}(x, y, h)=-\frac{E h}{1+\nu} \frac{\partial^{2} \bar{a}}{\partial \bar{x} \partial \bar{y}}=-\frac{E k h}{1+\nu} \frac{\partial^{2} a}{\partial x \partial y}
$$

where $s(x, y)$ and $a(x, y)$ are given by (3.37) and (3.41) respectively, and $\bar{s}(x, y)$ and $\bar{a}(x, y)$ are their dimensional counterparts.

It is a straightforward procedure, using the asymptotic properties (A.12) and (A.13), to show that

$$
\left(\frac{\partial^{2} s}{\partial y^{2}}+\nu \frac{\partial^{2} s}{\partial x^{2}}\right) \sim-\frac{\lambda^{2} f^{s}(\theta)}{K_{+}(\lambda \cos \theta)} \sqrt{\frac{(1-\nu)(3+\nu)}{2 \pi x}}, \quad y=0, x \rightarrow 0
$$

and

$$
\frac{\partial^{2} a}{\partial x \partial y} \sim \frac{i \lambda^{3} f^{a}(\theta)(1+\nu)}{\sqrt{2 \pi x(1-\nu)(3+\nu)} K_{+}^{a}(\lambda \cos \theta)}, \quad y=0, x \rightarrow 0 .
$$

It follows that

$$
\bar{\kappa}_{1}=\frac{E k h \lambda^{2} f^{s}(\theta) \sqrt{(1-\nu)(3+\nu)}}{\left(1-\nu^{2}\right) K_{+}(\lambda \cos \theta)}
$$

\footnotetext{
${ }^{3}$ The energy of the newly created crack surface per unit area.
} 
and

$$
\bar{\kappa}_{2}=-\frac{i E k h \lambda^{3} f^{a}(\theta)}{K_{+}^{a}(\lambda \cos \theta) \sqrt{(1-\nu)(3+\nu)}} .
$$

The stress intensity factors vary with current, frequency and the angle of the incident wave. Figure 4 shows a polar plot of $\left|\bar{\kappa}_{1} / E\right|$ and $\left|\bar{\kappa}_{2} / E\right|$ for a frequency of $500 \mathrm{~Hz}$ and three different values of current. The innermost curve corresponds to $J_{0}=$ $0 \mathrm{~kg}^{1 / 2} \mathrm{~s}^{-1} \mathrm{~m}^{-1}$ and the magnitude increases sequentially for $J_{0}=20000,50000 \mathrm{~kg}^{1 / 2} \mathrm{~s}^{-1} \mathrm{~m}^{-1}$.
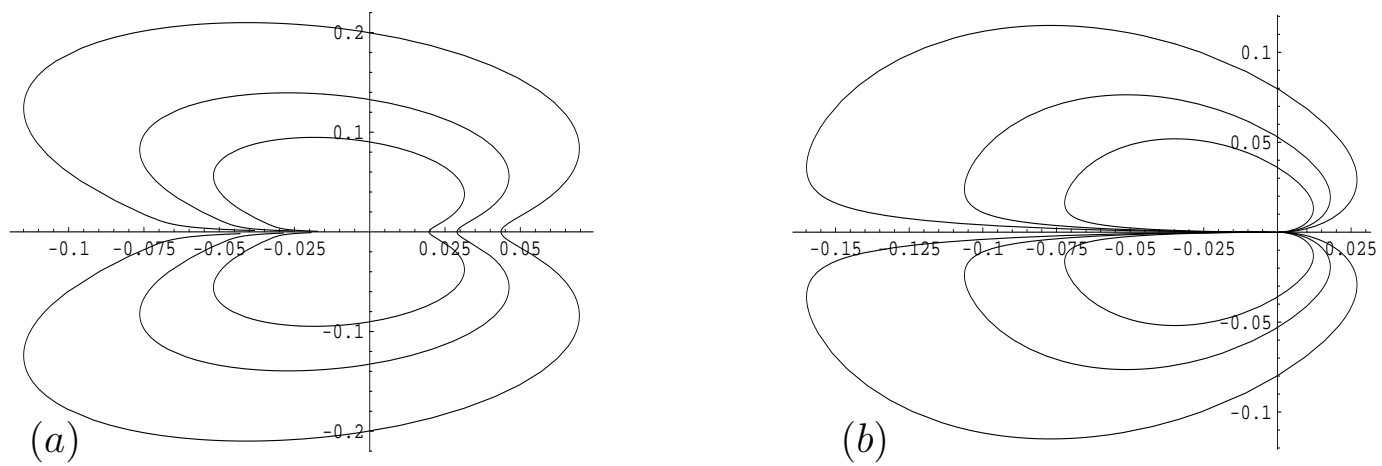

Figure 4: Polar plot of (a) $\left|\bar{\kappa}_{1} / E\right|$ for $J_{0}=0 \mathrm{~kg}^{1 / 2} \mathrm{~s}^{-1} \mathrm{~m}^{-1}$ (innermost curve), $J_{0}=20000$ and $J_{0}=50000$ (outermost curve); (b) $\left|\bar{\kappa}_{2} / E\right|$ for $J_{0}=0 \mathrm{~kg}^{1 / 2} \mathrm{~s}^{-1} \mathrm{~m}^{-1}$ (innermost curve), $J_{0}=20000$ and $J_{0}=50000$ (outermost curve).

Finally, the behaviour of the moments can be deduced as $\omega \rightarrow \infty$ (equivalently $\epsilon \rightarrow 0)$. It is found in this limit that $\lambda \rightarrow 1, \alpha_{2} \rightarrow 1$ and $\alpha_{1} \rightarrow i$. It is clear then that the stress intensity factors depend on $\omega$ only through the factor $k h$ and consequently are of order $\omega^{1 / 2}$ as $\omega \rightarrow \infty$. Thus,

$$
\frac{\bar{\kappa}_{1}}{E} \sim-\left(\frac{2 \rho}{D}\right)^{1 / 4} \frac{h^{5 / 4} \omega^{1 / 2} f^{s}(\theta) \sqrt{(1-\nu)(3+\nu)}}{K_{+}(\cos \theta)\left(1-\nu^{2}\right)}, \quad \omega \rightarrow \infty
$$

and

$$
\frac{\bar{\kappa}_{2}}{E} \sim\left(\frac{2 \rho}{D}\right)^{1 / 4} \frac{h^{5 / 4} \omega^{1 / 2} f^{a}(\theta)}{K_{+}^{a}(\cos \theta) \sqrt{(1-\nu)(3+\nu)}}, \quad \omega \rightarrow \infty
$$

\section{Discussion}

The plate equation (2.1) was derived in [1] strictly for a non-ferromagnetic material. For this reason, all numerical results presented both in [1] and this article have been generated for an aluminium plate. Furthermore, the reader is reminded that the quantity $J_{0}$ is proportional to the current density through the expression $J_{0}=$ $\sqrt{2 h \mu_{r} \mu_{0}} I_{0}$, in which $\mu_{0}, \mu_{r}$ are respectively the magnetic permeability in a vacuum and the relative magnetic permeability in the plate. Ambur et al. use currents up to $I_{0}=100 \sqrt{E} / h A m^{-2}$ for which $J_{0}=\sqrt{2 h \mu_{r} \mu_{0}} I_{0} \approx 930000 \mathrm{~kg}^{1 / 2} \mathrm{~s}^{-1} \mathrm{~m}^{-1}$. (As mentioned earlier, a different notation is used herein, where $I_{0}, J_{0}$ are equivalent to $J_{0}, \tilde{J}_{0}$ used in [1].) Thus, the choice of $J_{0}=0-50000 \mathrm{~kg}^{1 / 2} \mathrm{~s}^{-1} \mathrm{~m}^{-1}$ used in figures $2-4$ 
are well within the scaled current density range chosen in [1] and are more realistic than the upper values in Ambur et al.'s results.

It has been found that the phase speed of the edge wave is highly dependent on frequency and current. Two distinct asymptotic behaviours are observed depending on whether $\omega \rightarrow \infty$ with $J_{0}$ fixed or vice-versa; these limits correspond respectively to $\epsilon \rightarrow 0$ and $\epsilon \rightarrow \infty$. As $\omega \rightarrow \infty$ the phase speed grows as $O\left(\omega^{1 / 2}\right)$, whereas as $J_{0} \rightarrow \infty$ it tends to a constant value: $\omega h / \sqrt{3}$. The two stress intensity factors are both highly dependent on frequency, current and angle of incidence. Similar to the phase speed, they grow as $O\left(\omega^{1 / 2}\right)$ as $\omega \rightarrow \infty$.

To conclude, the results presented herein indicate that, although the current does alter the scattered field in a significant way, for physically reasonable current amplitudes it is unlikely to offer a mechanism for either suppressing crack growth, or for the continuous monitoring and detection of a new crack. It may, however, offer a useful means by which the flexural wave field can be localized or channelled along free edges.

\section{A Factorisation of the kernel}

A key feature of the kernel is that, using (4.43), it may be written in a form that is independent of $\gamma_{3}$. Thus,

$$
K(\alpha)=\frac{\sqrt{\gamma_{1} \gamma_{2}}}{\sqrt{2} \sqrt{1+\alpha^{2} /\left(\gamma_{1} \gamma_{2}\right)}}\left\{1+(1-\nu) \frac{2 \alpha^{2}}{\gamma_{1} \gamma_{2}}-\frac{\nu^{2} \alpha^{4}}{\gamma_{1}^{2} \gamma_{2}^{2}}\right\} .
$$

It is immediately apparent that $\gamma_{1}$ and $\gamma_{2}$ appear in this expression only as a product. Further, this product is exactly equivalent to the product of two simpler square root functions: $\eta_{1}(\alpha)$ and $\eta_{2}(\alpha)$. That is,

$$
\gamma_{1} \gamma_{2}=\left(\alpha^{4}+\epsilon^{2} \alpha^{2}-1\right)^{1 / 2}=\left(\alpha^{2}-\alpha_{1}^{2}\right)^{1 / 2}\left(\alpha^{2}-\alpha_{2}^{2}\right)^{1 / 2}=\eta_{1} \eta_{2}
$$

and so the branch-points are easily seen to be

$$
\alpha_{1}=i \sqrt{\frac{\sqrt{\epsilon^{4}+4}+\epsilon^{2}}{2}}, \quad \alpha_{2}=\sqrt{\frac{\sqrt{\epsilon^{4}+4}-\epsilon^{2}}{2}} .
$$

In order to facilitate the product factorisation, it is expedient to rearrange the kernel in terms of the function $L(\alpha)$ where $L(\alpha) \sim 1$ as $|\alpha| \rightarrow \infty$. Thus,

$$
K(\alpha)=\frac{\sqrt{\eta_{1} \eta_{2}}(1-\nu)(3+\nu)\left(\alpha^{2}-\alpha_{e d}^{2}\right)\left(\alpha^{2}-\alpha_{e v}^{2}\right)}{2\left(\alpha^{2}-\alpha_{1}^{2}\right)\left(\alpha^{2}-\alpha_{2}^{2}\right)} L(\alpha)
$$

where

$$
\begin{aligned}
L(\alpha)= & \frac{\sqrt{2}\left(\alpha^{2}-\alpha_{1}^{2}\right)\left(\alpha^{2}-\alpha_{2}^{2}\right)}{(1-\nu)(3+\nu)\left(\alpha^{2}-\alpha_{e d}^{2}\right)\left(\alpha^{2}-\alpha_{e v}^{2}\right) \sqrt{1+\alpha^{2} /\left(\eta_{1} \eta_{2}\right)}} \\
& \times\left\{1+(1-\nu) \frac{2 \alpha^{2}}{\eta_{1} \eta_{2}}-\frac{\nu^{2} \alpha^{4}}{\eta_{1}^{2} \eta_{2}^{2}}\right\} .
\end{aligned}
$$

Note that the terms preceding $L(\alpha)$ in (A.4) can be factorised by inspection, and the behaviour of $L(\alpha)$ as $|\alpha| \rightarrow \infty$ is expediently chosen to allow it to be factorised using 
Cauchy's integral formula. Further, $L(\alpha)$ is defined so that it does not have zeros at $\alpha= \pm \alpha_{e d}$ or $\alpha= \pm \alpha_{e v}$ but it does contain branch-points at $\alpha_{1}$ and $\alpha_{2}$. In fact, $L(\alpha)$ is a function of the combined branch-cut term $\eta_{1} \eta_{2}$ (A.2), which conveniently can be chosen to have finite cuts lying between $\alpha_{2}$ and $\alpha_{1}$, and between $-\alpha_{2}$ and $-\alpha_{1}$. That is, for both functions $\eta_{1}$ and $\eta_{2}$ the upper half plane cuts are chosen to lie along the straight line that connects the point $\alpha_{2}$ to $\alpha_{1}$ and continue into the second quadrant of the complex plane. Thus, the branch-cuts overlap and lie at an angle $\psi$ to the real line (see figure 5) where

$$
\psi=\pi-\arctan \left(\frac{\left|\alpha_{1}\right|}{\alpha_{2}}\right)=\pi-\arctan \left(\sqrt{\frac{\sqrt{\epsilon^{4}+4}+\epsilon^{2}}{\sqrt{\epsilon^{4}+4}-\epsilon^{2}}}\right) .
$$

(Note that this quantity is not related to the polar angle of section 4.1.) Similarly, the overlapping lower half plane cuts lie along the line joining $-\alpha_{2}$ to $-\alpha_{1}$ and continue into the fourth quadrant. A consequence of this choice of branch-cuts is that for quantities such as $\eta_{1} \eta_{2}$ and $\eta_{1} / \eta_{2}$ the overlapping sections of the branch-cuts "cancel", and so, as mentioned, these quantities have only finite length cuts lying along the line segments joining $\pm \alpha_{2}$ and $\pm \alpha_{1}$. Having defined the branch-cuts, the appropriate sheet of the Riemann surface branches is now chosen such that $\eta_{1}(0)=-i \alpha_{1}$ and $\eta_{2}(0)=-i \alpha_{2}$.

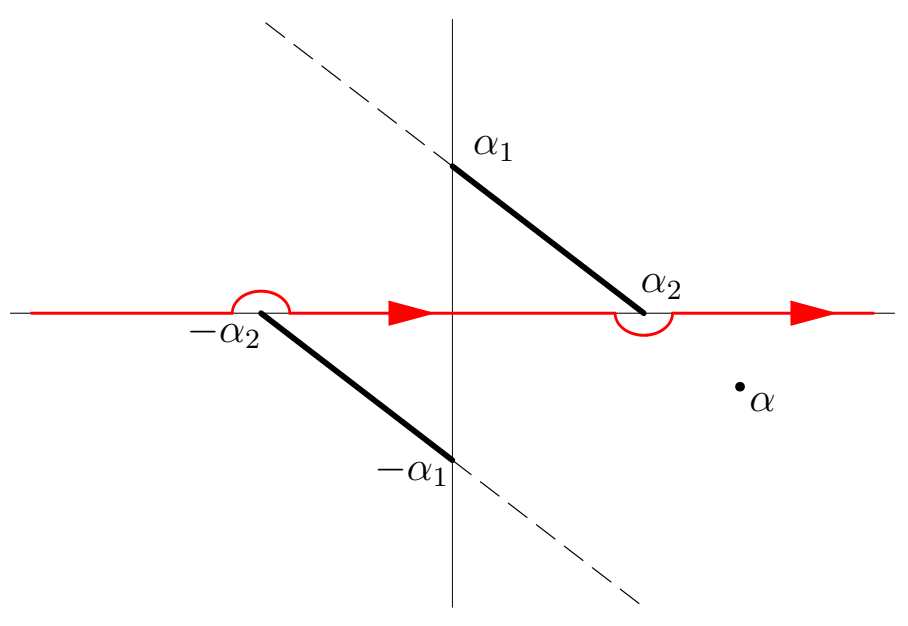

Figure 5: The overlapping branch-cuts for $\eta_{1}, \eta_{2}$ and the path of integration for $P_{-}(\alpha)$.

Cauchy's integral formula can now be exploited to obtain the product factors of $L(\alpha)$. The lower function is defined [14] as

$$
L_{-}(\alpha)=\exp \left\{-\frac{1}{2 \pi i} \int_{C} \log [L(\zeta)] \frac{d \zeta}{\zeta-\alpha}\right\}=\exp \left\{-\frac{1}{2 \pi i} P_{-}(\alpha)\right\},
$$

where $\alpha$ lies in the lower half plane and the path of integration is indented above (below) any singularities on the negative (positive) real line, see figure 5 . On deforming the path of integration into the upper half plane and noting that there is no contribution from the arc at infinity, it is found that

$$
P_{-}(\alpha)=\int_{\alpha_{2}}^{\alpha_{1}} \log [g(\zeta)] \frac{d \zeta}{\zeta-\alpha}
$$


where $g(\zeta)=L^{u}(\zeta) / L^{\ell}(\zeta)$ and the superscripts $\ell$ (lower) and $u$ (upper) indicate on which side of the cut the function must be evaluated. On noting that

$$
\eta_{2}^{\ell}=-\eta_{2}^{u}
$$

$g(\zeta)$ can be expressed as

$$
g(\zeta)=\frac{\sqrt{\eta_{1} \eta_{2}-\alpha^{2}}\left\{\eta_{1}^{2} \eta_{2}^{2}+2(1-\nu) \alpha^{2} \eta_{1} \eta_{2}-\alpha^{4} \nu^{2}\right\}}{\sqrt{\eta_{1} \eta_{2}+\alpha^{2}}\left\{\eta_{1}^{2} \eta_{2}^{2}-2(1-\nu) \alpha^{2} \eta_{1} \eta_{2}-\alpha^{4} \nu^{2}\right\}}
$$

where here and henceforth $\eta_{2}$ will be taken to denote $\eta_{2}^{u}$. Thus,

$$
\begin{aligned}
K_{-}(\alpha)= & \sqrt{\frac{(1-\nu)(3+\nu)}{2}}\left(\sqrt{\eta_{1} \eta_{2}}\right)_{-} \frac{\left(\alpha-\alpha_{e d}\right)\left(\alpha-\alpha_{e v}\right)}{\left(\alpha-\alpha_{1}\right)\left(\alpha-\alpha_{2}\right)} \\
& \times \exp \left\{-\frac{1}{2 \pi i} \int_{\alpha_{2}}^{\alpha_{1}} \log [g(\zeta)] \frac{d \zeta}{\zeta-\alpha}\right\},
\end{aligned}
$$

where the cut function, with cuts shown in figure 5 , is given by

$$
\left(\sqrt{\eta_{1} \eta_{2}}\right)_{-}=e^{i(\psi / 2-\pi / 4)}\left(e^{i(\pi-\psi)}\left(\alpha-\alpha_{1}\right)\right)^{1 / 4}\left(e^{i(\pi-\psi)}\left(\alpha-\alpha_{2}\right)\right)^{1 / 4} .
$$

Similarly,

$$
\left(\sqrt{\eta_{1} \eta_{2}}\right)_{+}=e^{i(\psi / 2-\pi / 4)}\left(e^{-i \psi}\left(\alpha+\alpha_{1}\right)\right)^{1 / 4}\left(e^{-i \psi}\left(\alpha+\alpha_{2}\right)\right)^{1 / 4} .
$$

Note that

$$
K_{-}(\alpha) \sim \sqrt{\frac{(1-\nu)(3+\nu)}{2}} \alpha^{1 / 2} e^{i \pi / 4}, \quad \alpha \rightarrow \infty,
$$

where the branch-cut for $\alpha^{1 / 2}$ lies in the upper half plane, whilst the anti-symmetric kernel is given by $K^{a}(\alpha)=\gamma_{1} \gamma_{2} K(\alpha)$, and so it follows that

$$
\begin{aligned}
K_{-}^{a}(\alpha) & =-\sqrt{\frac{(1-\nu)(3+\nu)}{2}} \frac{\left(\alpha-\alpha_{e d}\right)\left(\alpha-\alpha_{e v}\right)}{\left(\sqrt{\eta_{1} \eta_{2}}\right)_{-}} \exp \left\{-\frac{1}{2 \pi i} \int_{\alpha_{2}}^{\alpha_{1}} \frac{\log [g(\zeta)]}{\zeta-\alpha} d \zeta\right\} \\
& \sim \sqrt{\frac{(1-\nu)(3+\nu)}{2}} \alpha^{3 / 2} e^{3 i \pi / 4}, \quad \alpha \rightarrow \infty
\end{aligned}
$$

\section{References}

[1] Ambur, D.R., Hasanyan, D., Librescu, L. and Qin, Z. Diffraction of harmonic flexural waves in a cracked elastic plate carrying electrical current. Proceedings of the Royal Society of London, A461, 3542-3560 (2005).

[2] Konenkov, Y.K. A Rayleigh-type flexural wave. Soviet Physics Acoustics. 6, 122$123(1960)$.

[3] Lawrie, J.B. and Kaplunov, J.D. Edge waves and resonance on elastic structures: an overview. Mathematics and Mechanics of Solids. In press. 
[4] Ishlinskii, A.Y. On a limiting process in the theory of the stability of elastic rectangular plates. Dokl. Akad. Nauk SSSR 95(3), 477-479 (1954).

[5] Sinha, B.K. Some remarks on propagation characteristics of ridge guides for acoustic waves at low frequencies. Journal of the Acoustical Society of America, 56, 16-18 (1974).

[6] Thurston, R.N. and McKenna, J. Flexural acoustic waves along the edge of a plate. IEEE Transactions on Sonics and Ultrasonics, 21, 296-297 (1974).

[7] Norris, A.N. Flexural edge waves. Journal of Sound and Vibration. 171, 571-573 (1994).

[8] Norris, A.N. and Wang, Z. Bending wave diffraction from strips and cracks on thin plates. Quarterly Journal of Mechanics and Applied Mathematics, 47, 607627 (1994).

[9] Thompson, I., Abrahams, I.D. and Norris, A.N. On the existence of flexural edge waves on thin orthotropic plates. Journal of the Acoustical Society of America, $112,1756-1765$ (2002).

[10] Fu, Y. Existence and uniqueness of edge waves in a generally anisotropic elastic plate. Quarterly Journal of Mechanics and Applied Mathematics. 56, 605-616 (2003).

[11] Shindo, Y., Ogihara, A. and Horigudi, K. Dynamic bending of a magnetically saturated plate with a through crack in a uniform magnetic field. Theoretcial Applied Mechanics 49, 69-81 (2000).

[12] Graff, K. Wave motion in elastic solids, Dover Edition, Dover Publications Inc., New York, 1991.

[13] Lawrie, J.B. and Abrahams, I.D. A brief historical perspective of the WienerHopf technique. Journal of Engineering Mathematics, 59, 351-358 (2007).

[14] Noble, B. Methods based on the Wiener-Hopf Technique, Second Edition, Chelsea Publishing Company, New York, 1988.

[15] Thompson, I. and Abrahams, I.D. Diffraction of flexural waves by cracks in orthotropic this elastic plates. I Formal solution. Proceedings of the Royal Society of London, A461, 3413-3436 (2005).

[16] Thompson, I. and Abrahams, I.D. Diffraction of flexural waves by cracks in orthotropic this elastic plates. II Far field analysis. Proceedings of the Royal Society of London, A463, 1615-1638 (2007).

[17] Thompson, I. An improved uniform approximation for diffraction integrals. Proceedings of the Royal Society of London, 462, 1341-1353 (2006).

[18] Zernov, V. and Kaplunov, J. Three dimensional edge-waves in plates. Proceedings of the Royal Society of London, A464, 301-318 (2008). 
[19] Zucchini, A., Hui, C.T.and Zehnder, A.T. Crack tip stress fields for thin, cracked plates in bending, shear and twisting: A comparison of plated theory and threedimensional elasticity theory solutions. International Journal of Fracture. 104, 387-407 (2000).

[20] Zehnder, A.T. and Viz, M. Fracture mechanics of thin plates and shells under combined membrane, bending and twisting loads. Applied Mechanics Reviews. 58, 1-43 (2005) .

[21] Young, M.J. and Sun, C.T. Cracked plates subjected to out-of-plane tearing loads. International Journal of Fracture. 60, 1-18 (1993). 